\title{
LA POETICA DI ANTONIO FOGazzaro
}

\author{
Carolina Massi Albanese \\ Universidade Federal do Paraná
}

\begin{abstract}
RIASSUNTO
Le opere del Fogazzaro traboccano nell'autobiografismo, che non è confessione dolente, ma si, rappresentazione delle perplessità, delle esperienze morali e religiose dell' autore. In esse vi è una proposta religiosa ed anche una proposta poltica che, scaturite da una congiunzionc ibrida dell'ideale e del reale, si revelano contaminate e confuse.

Questi "principi" fogazzariani, tuttavia, sotto una prospettiva diversa inquadrata nei tempi e nella realtà storica del momento, danno una validità al proposito di predicazione religiosa e politica.

I protagonisti dei romanzi, esseri fuori della norma, sono in complesso, piuttosto scialbi, sofisticati e staccati dal contesto poetico perchè soverchiati dalla presenza dell'autore. L'autore, difatti, ripropone il protagonista anteriore con alcune pcche variazioni e con nome differente: il personaggio principale non è altro che il personaggio nel quale l'autore vuol rappresentarsi con tutti i suoi conflitti, i suoi contrasti fra la terra e il clelo.
\end{abstract}

Nella narrativa italiana della fine dell'ottocento occupa un gran posto il vicentino Antonio Fogazzaro.

Nacque un Venerdi Santo, il 25 marzo 1842, da Mariano, fervente patriota e profondamente religioso, e da Teresa Barrera, una borghese, figlia di un architetto della Valsolda, trasferito a Vicenza.

Fin dalla sua infanzia il Fogazzaro andò spesso a Villeggiare ad Oria, nella Valsolda, terra d'origine della madre; questa valle, sulla riva del lago di Lugano, rimarrà sempre 
nell'animo dello scrittore, influenzerà profondamente la sua ispirazione e da essa trarrà molti motivi di sviluppo dei suoi romanzi e delle sue poesia.

\section{II pensiero del Fogazzaro.}

Il Fogazzaro visse negli anni in cui era ancora vivo il ricordo del Risorgimento, periodo in cui si lavorava all'assestamento del nuovo Regno ed incominciava il fermento delle riforme sociali.

Egli non fu un politico nè un sociologo in senso stretto, anche se si interessò formalmente dei problemi del suo tempo.

Le idee politiche, sociali e reiigiose vengono affrontate nei romanzi Il Santo e Danicle Cortis, ma nonostante il desiderio di rinnovamento e di umanità, il Fogazzaro non accettò in pieno il programma socialista, nè la collaborazione con $i$ socialisti. Cercò di sottolineare le divergenze tra il programma cristiano e quello socialista, precisando le sue concezioni su Il Giornale d'Italia del 5 agosto 1908: "Il primo ha radice nell'amore dato doverosamente a tutti gli uomini senza clistinzione, nell'amore che vuole il bene dei ricchi e dei poveri, un bene di ordine morale cui pone supremo un bene contrario e superiore a tutte le cupidigie, a quelle del povero come a quelle del ricco; il secondo, quando è ottimo, ha radice in un desiderio di giustizia, senza dubbio, ma pone supremo un bene di ordine materiale e non ha substrato di amore" 1 (p. 505).

L'unità nazionale aveva impegnato le risorse di tutta una generazione: l'aspettativa ingenua dei più pensava che la trasformazione politica avrebbe risolto in parte $i$ problemi economici e sociali, ma i risultati si dimostrarono inferiori alle speranze e l'intrinseca debolezza del Paese costitui un'amara delusione per quanti si erano formati alla scuola degli eroici miti nazionali.

L'aspetto negativo avrebbe potuto essere superato o idealizzato dalla letteratura e dalle arti.

1 I branl c concet:i riportat nol prcserte iarcro scno :rat!l dalla raccolts compleia Vita e opero di Antonio Fosazzro, 3 cura di Plero Nardi. Milsno, Mondadorl. 1931. 12 ;. Cl lliniticremo. pertanto, a cilaze jo:o ll numero della panina. 
Il Fogazzaro prese parte a quesio movimento di riforma accettando l'ideologia del neoguelfismo che cercava di adeguarsi agli ideali umanitari e socialisti. Ma, mentre Pascoli aveva abbracciato il nucleo delle idee socialiste ed operò da socialista, il Fogazzaro non si immedesimò mai nei problemi economici e sociali delle masse proletarie, tanto che il Donadoni asserisce che "il mondo degli umili ha dato alla produzione fogazzariana figure prettamente grottesche e riempitive: il servo esiste in relazione al padrone".

Il Fogazzaro accetta in parte la politica cavouriana ribadendo in Daniele Cortis la distinzione tra i due poteri, temporale e spirituale. La carriera politica di Daniele gli offre l'occasione di esporre le sue idee di una monarchia forte (quasi una dittatura) che, fuori degli appoggi parlamentari, sia atta per ardite riforme sociali.

Al carattere politico s'innesta la concezione religiosa. Durante il periodo di indifferentismo religioso, la religiosità del Fogazzaro era svaporata in una visione animista e panteista. La conversione si svolse in circostanze, le quali, poco credito davano ad una stabilità e nasceva da una fantastica sofistificazione, nonchè da una sentimentalità poetica e vaga. Fu questo il momento di incontro del Fogazzaro con i riformisti cattolici e lui, pur accettandone le comuni ispirazioni, se ne distaccò in nome della scienza e della modernità.

Gli studi scientifici e filosofici lo portarono ad accettare in parte le teorie del trasformismo. Fogazzaro riteneva che l'idea dell'evoluzione della specie, sostenuta dal Darwin, non solo era contraria alla tesi dimostrativa dell'esistenza di Dio, ma ne rappresentava la più felice dimostrazione scientifica. La creazione, riteneva il Fogazzaro, lungi dall'essersi risolta in un unico atto creativo, "si realizzerebbe lungo il processo storico con una continuità pari a quella della vita degli esseri". (p. 29).

Si scontrò con il pensiero tradizionalistico ed intransigente della Chiesa in quanto il Fogazzaro, pur accettando l'essenza del cattolicesimo, desiderava riformare la parte istituzionale della Chiesa stessa, togliere allo scolasticismo le antiche sovrastrutture, ringiovanire e ridestare, attraverso la nuova teoria, il sentimento religioso che stava attraver-

2 DoNadoNi. Eucento. Antonio Fouazzaro. Nanoll. Perrella. 1913. p. 118. 
sando un periodo di profonda crisi spirituale. Siamo in un momento di crisi nella ricerca di ideali ed il Fogazzaro avverte la necessità di un principio metafisico che non riesce a chiarire a se stesso. La sua è un'analisi psicologica minuta e sofferente, uno scendere nel fondo della coscienza ed aggirarvisi con profondo tormento senza mai trovare l'appagamento di cui aveva tanto bisogno. Per questo desiderio di ricerca, di sofferenza e di angoscia, possiamo avvicinare il Fogazzaro al decadantismo. Il Fogazzaro visse il dramma dell'angoscia non a livello logico e di interpretazione filcsofica, ma a livello sentimentale e poetico. La sua indagine verso i problemi morali e religiosi non scaturiva da una convinzione lineare $e$ sostenuta su base filosofiche, per cui il desiderio di analizzare e scrutare la parte più segreta dell'animo umano rappresentava per lui uno sconfinamento verso orizzonti indimostrabili. Cercò di concretizzare la sua ideologia con Il Santo, nel quale avrebbe voluto raffigurare il modello di un santo moderno, operante a ravvivare il sentimento religioso e ad ammodernare la Chiesa. Ma proprio questo santo è il personaggio più ambiguo della sua opera, per la impossibilita dello scrittore di liberarsi dalle inquietudini, da una sensualitá tanto più acre quanto più trattenuta. La condanna della Congregazione dell'Indice dell'opera Il Santo, in cui il Fogazzaro aveva affrontato sentimentalmente la nuova corrente modernista, svaluto immediatamente le manifestazioni polemiche del ro. manziere e del pensatore.

Il Fogazzaro si sottomise senza riserve e pubblicamente, scatenando una tempesta tra quanti vedevano nella sua condotta una rinuncia alla propria libertà intellettuale e quanti, al contrario, vi videro una buona dose di coraggio morale e di nobile fedeltà ad un principio superiore.

La partecipazione fogazzariana all'attività modernista fu quindi limitata, sentendosi il Fogazzaro staccato nel profondo del sentimento dalle disquisizioni aride quanto erudite di alcuni; infatti egli sentì che gli schemi logici della filosofia non ingrandivano la fede e che l'integrita spirituale era minorata dall'analisi e dalla ragione.

Il lato più originale della teoria fogazzariana è quello dell'arte come espressione di spilitualità umana, in cui l'estetica evoluzionistica trova spazio per spiegare la bellezza dell' 
espressione e la concezione dell'amore come elevazione morale. Egli si schiera con quegli artisti che combattono l'arte verista con lo spiritualismo e tende a valorizzare l'arte come aspirazione individuale, e il pueta rappresenta l'incarnazione della grandezza spirituale e morale.

La poesia è per il Fogazzaro desiderio sentimentale che domina l'animo e 10 rende incapace di riflettere. Egli esprime la necessità di identilicare la poesia con l'amore, poichè dal soffio dell'amore emana la virtù e la felicità della poesia. Quindi possiamo dire che per il Fogazzaro la poesia è soprattutto fremito sentimeintale e l'amore fremito presessuale.

Nella novella in versi Miranda si avverte il carattere dell'amore, così come nel proemio alle Ascensioni Umane, puntualizzando la tendenza dello spirito a sovrapporsi all'instinto sessuale e a signoreggiare sull'intelligenza.

I personaggi fogazzariani amano guardarsi e contemplare i propri sentimenti, non soffrono vere passioni. Gli amanti amano compiangersi, non hanno la forza di protestare e tanto meno di uccidersi.

L'erotismo è uno stato d'animo costante di cui il Fogazzaro non si libererà mai; basta pensare che nei romanzi scritti in età matura prende forma la figura del vecchio che ama riamato una giovane.

L'amore in Fogazzaro desta per antitesi il senso della morte, sentita in quel che ha di sereno, di purificatore, ma anche in quello che ha di tragico, illogico e ingiusto.

Il Fogazzaro è in uno stato perpetuo di conato verso idealità transcendenti, ma rimane sempre ancorato alla realità del suo mondo morale, come non sa mai uscire dalla realtà del mondo sociale in cui vive.

Il Fogazzaro si rivela artista del paesaggio, di montagna, di laghi, di fiumi, di cime nevose, trasfondendo nella natura lo stato d'animo del personaggio, creandone un tutt'uno come nell'espressione: "Mi abbraccio ad una magnolia, le chiedo che ha, che mai sia. Giù per i rami e le foglie per la mia fronte e le ciglia, il pianto tacito va" (p. 258).

Nella Valsolda riscontra l'animazione della natura che rivela sia all'autore che al lettore la presenza di una forza sovrumana. 
Anche in Fascino la luna sale in cielo da lontani orizzonti e il velo della notte avvolge il lago e le valli segrete, la cascata urla fra dirupate sponde e il viandante si sente colpito "da una voce che viene da una creatura che lo imita nel seno di occulte fonti, tra golfi cupi, tra spume ed onde in cui essa vive" (p. 260).

L'influsso della poesia nordica è evidente nelle opere del Fogazzaro, ma egli è riuscito a darne una interpretazione personale, creando delle sinfonie in cui $i$ temi paesistici si succedono quasi in una sintesi esemplificatrice, pur con le loro vibrazioni ed i loro sottintesi umani, anche se durante la narrazione l'aspetto naturalistico è velato dalle passioni dei personaggi con una penetrazione cosi intensa da creare una specie di paesaggio allegorico.

A volte il Fogazzaro va alla ricerca di espressioni indefinite per meglio evidenziare la sensazione del vago e del mistero come si nota in Sllenzio: "da occulta parte dentro a me l'albore dimana dell'eterno. Il mio pensier vi si sprofonda, naviga oltre i liti di ogni cosa creata e là si solve com'esta bolla che gorgoglia e tace a fior dell'acque vitree, Iontano" (p. 262).

Possiamo definire il Fogazzaro come poeta delle descrizioni di paesaggi inquieti, delle impressioni sfumate, degli stati d'animo dolorosi, dove la tecnica dell'espressione rivela stati psicologici, sensazioni immediate in cui il paesaggio sfuma in un'aspirazione musicale e sognante. Egli si orienta così verso la musica creando degli stati d'animo malinconici, popolati di ricordi affettivi e di evocazioni femminili. Rivive coil nostalgia quella parte del lago di Lugano in cui era solito villeggiare, quella parte di Valsolda che avrà una notevolissima importanzza nello sviluppo successivo della sua arte. Infatti questi paesaggi non solo daranno lo spunto a molte sue liriche, ma diventeranno anche l'ambiente nel quale il Fogazzaro collocherà l'azione dei suoi romanzi. E stato bene osservato dal Donadoni che nei romanzi del Fogazzaro "esistono giardini ordinati con arte ma non si avverte l'amore per la campagna, per la vita e per il lavoro nei campi" 3 .

Il Croce ne ha dato un'interpretazione particolare, rivelando l'aspetto incestuoso della fantasia fogazzariana che con-

3 DONADONI. D. 119. 
cepisce la natura come peccato e, a proposito dell'ultimo romanzo, scriveva che in Lella "continuano i velligimenti, le ebrezze e gli spasimi del senso" 4 .

Fogazzaro attua degli esperimenti metrici e stilistici cercando di tradurre metricamente le impressioni che gli deri. vano dalla musica. "La musica migliore genera in molti e anche in me ombre vane, per così dire di sentimenti, gioia e dolore senza causa, desiderio, sgomento, pietà senza oggetto, baldanze superbe che cadono con l'ultima nota, violenti impulsi ad impossibili azioni" (p. 42). (La musica) suggerisce pure confuse immagini alla fantasia... "io per parte mia ho talvolta cercato di consolarmene immaginando o scrivendo cio che lingua ignota potrebbe forse significare, ctò che vi potrebbe essere al di là della porta impenetrabile, le cause arcane di quei sentimenti la cui sola ombra mi commuoveva tanto" (p. 43).

\section{Le principali opere del Fogazzaro.}

Valsolda (1876)) (p. 256-78)

E una raccolta di poesie composte con metri diversi e pervase di allegorismo, in quanto il Fogazzaro interpreta la realtà con atteggiamento allegorico, adombrandola e quasi velandola con immagini che esprimono un mondo diverso e autonomo. Tali liriche sono inoltre caratterizzate dal simbolismo, dal musicalismo e dal panteismo, perchè il poeta tende ad esprimersi con simboli, ad esaltarne ogni aspetto musicale e ad identificare Dio con la natura.

Unico punto di riferimento dell'opera è il paesaggio della Valle del Soldo, piccolo fiume che getta le sue acque nella parte orientale del lago di Lugano. $E$ tale paesaggio è caro all'animo del poeta, non solo perche è la terra natale della famiglia di sua madre, ma soprattutto perchè è il luogo dove, durante la su afanciullezza ed anche dopo, si recava a trascorrere lietamente le sue vacanze autunnali e in cui ha tanto sognato e vissuto in spirituale corrispondenza con quanto di bello ha la natura nei suoi aspetti più semplici e più veri. Sente il poeta, quasi in perfetta sintonia, le voci che erom-

4 Croce. Bencdetto. Anton!o Fogazznro. In: Itail=. Bar!, Laterzn. 1847. p. 302 
pono misteriose dalle parti più nascoste di questo paesaggio di fronte al quale egli è come incantato e tutto proteso a recepime, nell'attimo fuggente, ogni fremito, ogni dolcezza.

Le poesie di questa raccolta che destano maggiore interesse sono: Silenzio, Il ritorno dal lavoro, A sera, Novissima verba, Dramma notturno.

La critica accolse l'opera con una certa riserva, ed $\dot{e}$ autorevole, a proposito, il giudizio del Croce che mette in risalto l'aspirazione del poeta ad esprimere compiutamente quanto avverte con la sua sensibilità di artista, ma questo suo desiderio non riesce a manifestarsi che approssimativamente poichè in esso "vibra l'emozione più che la meditazione e raramente le immagini si delineano con contorni precisi, ma assumono forme vaghe ed indistinte"."

Il Donadoni, da parte sua, ci dice che la poesia più bella è A sera perchè in essa il poeta è riuscito ad esprimere veramente tutto se stesso, "la sua religiosità intesa non solo come fede, ma anche come completo abbandono, il senso dell'ar. cano, non solo concepito come espresione di dolore, ma come tormento di esausto amore"."

\section{Malombra (1881)}

Fu il primo romanzo del Fogazzaro. Questo libro (p. 502725 ) presenta un intreccio complesso e consta di tre racconti, uno alluccinante e violento, uno idillico e riposante, uno comico e noioso, non bene fusi tra loro, anzi sembra che ognuno si contrapponga agli altri.

Il racconto è imperniato sulle allucinanti vicente attraverso le quali brucia gli ultimi anni della sua breve vita una giovane bella e orgogliosa, Marina di Malombra, la quale in un cassetto di un "secretaire" della sua camera da letto, ritrova un guanto, una ciocca di capelli ed uno specchio, insieme ad uno scritto di una sua antenata, Cecilia, tenuta prigioniera in quella stanza da un marito geloso. Poche ma impressionanti le parole contenute nello scritto: "Tu che ti troverai a leggere queste parole, riconosci in te la mia anima 
sventurata" (p. 521). Marina, nel leggere queste parole resta suggestionata, anzi ne è addirittura sconvolta. Parole che sembrano una rivelazione $e$ in lei subentra la convinzione, che si fa ossessione, di incarnare l'anima dell'antenata. Crede di dover rivivere le vicende della morte e vede nello zio, il persecutore. Sente in sè la necessità di vendicare la disgraziata con l'uccisione dello zio, dal quale si crede perseguitata. In Corrado Silla, scritorello fatuo e debole che lei ammira e fa da segretario allo zio, vede reincarnato il giovane che sospirò per la infelice Cecilia Corrado sente per Marina solo un'accensione sensuale dalla quale tenta di liberarsi. Quando però Marina lo mette alla prova, egli scatena senza volerlo la follia che già covava in quell'anima e muore per mano di lei.

Il Fogazzaro avvolse la storia di una suggestiva magia che ricorda le saghe nordiche: la natura, il lago ed il palazzo in un atmosfera di romanticismo ignoto alla letteratura italiana. Gli elementi in cui vivono i personaggi sembrano come stregati da una forza invincibile e misteriosa. C'è qualcosa di alluccinante nella morte dello zio e di Silla, uccisi da Marina, così come nella fine della protagonista, che è il personaggio misterioso e inquietante.

A questa atmosfera di saga nordica si contrappone la storia serena di un altro gruppo di personaggi: don Innocente, Edith e suo padre Steinegge, ex capitano austriaco, che la figlia riporta alla fede perduta. La ragazza rinuncia all'amore nato per Corrado Silla pur di assolvere pienamente la sua misione. Il Fogazzaro chiamo Edith "reazione della coscienza e del sentimento religioso" (p. 578) e ne volle fare una contrapposizione ideale al personaggio di Marina.

Questa fidanzata ideale di Corrado Silla, il quale vorrebbe rifugiarsi nell'amore puro di lei per riscattare quello peccaminoso per Marina, è poco indagata dal poeta che ne confessava di non averla amata per non addolcirne $i$ contorni rigidi.

La terza storia, la nota comica del romanzo, è la vicenda della contesse Fosca e del figlio Nepo che sono vere macchiette caricaturali più che personaggi. 
Il tentativo di fusione di tragico sentimentale e comico, che si trova in Malombra, stabilisce, per cosi dire, quello che sarà un pò la maniera del raccontare in altri romanzi. In Malombra questa fusione è piuttosto scomposta ma più istintiva. Vi si inizia anche la confessione autobiografica. Corrado Silla, infatti, è il Fogazzaro, il quale sentiva o credeva di sentirsi nella giovinezza un mistico snervato dalla sensualità. Le st:e lotte non sono che suggestioni per evitare gli esatti termini del suo problema morale. E' il lirismo del Fogazzaro personificato in un personaggio, Corrado Silla, un sognatore, incapace di attaccarsi alla vita e vivente un mondo autonomo. Ha bisogno dell'amore per vivere, o meglio, per lasciarsi vivere. Eppure, nello spostare in Silla la sua discontinuita psicologica del periodo che attraversa, accentua i punti negativi della sua anima, quasi per compiacersi del desiderio di autolesionismo. In generale il personaggio è fiacco, ambiguo. Anche Marina che doveva essere una creatura eccezio. nale, finisce per diventare un impasto di orgoglio, sensualità, morbosità, una nevrotica. Malombra è senza dubbio la più sensuale delle eroine del Foga:szaro.

Nei personaggi minori c'è più schiettezza, perchè non sovraccaricati di interessi personali che lo scrittore voleva affermare nelle sue opera.

Anche se la critica addolorò il Fogazzaro, questo romanzo creò la fama negli ambienti letterali italiani c stranieri. Il successo di pubblico fu tale che in tre mesi soltanto si esauri la prima edizione del libro.

Quest'opera uscì nel 1881, anno di pubblicazione dei Malavoglia del Verga, che rappresenta il capolavoro del Verismo italiano. Verga lodò molto l'opera fogazzariana sia pure cosi diversa dalla propria vocazione letteraria.

La critica successiva, che ha avuto tempo per esaminare Malombra con più pacatezza, ne ha evidenziato più i pregi che i difetti. Il Momigliano ha definito Malombra "una creazione magica e il tentativo meraviglioso di esprimere l'inesprimibile e la riuscita esperienza di tradurre le voci dell'inconscio":

7 Momigliano, Attilio. Antonio Fozszaro. In: - - Elzeviri. Flrenze. Le Monnler. 2945. D. 218. 


\section{Daniele Cortis (1885)}

Il Fogazzaro, mentre in Malombra aveva cercato di rappresentare la lotta tra la carne e lo spirito, argomento chiave della sua opera. in Daniele Cortis (p. 1130-375) cerca di evidenziare la lotta tra la passione e il dovere, tra il senso e la morabilità. Daniele, l'uomo politico, rappresenta non tanto il personaggio delln passione amorosa quanto il protagonista dell'icieologia politica.

La carriera politica di Daniele offre all'autore l'occasione di esporre le sue idee di una monarchia forte (quasi una dittatura) che, fuori degli appoggi parlamentari, sia atta per ardite riforme sociali.Tuttavia il Fogazzaro non è.mai riuscito ad inserire il personaggio nella realtà storica, in quella realtà del XIX s̈ecolo che vedeva ormai l'inserimento delle masse proletarie.

Daniele Cortis, protagonista incerto e reticente nei suoi moti psicologici, trova la sua fonte di coraggio in una nobile anima alla quale è legato da affinità sentimentali: Elena Carrer. Questa donna, creatura del sentimento che vive la realtà attraverso la propria sensibilità, non riesce a creare un dialogo con il marito che dedito al gioco e agli affari, la trascura. Elena si sente quiridi attratta dal cugino Daniele e conscia della passione che la tormenta, lascia Roma per non cadere in adulterio. Elena infine segue il marito in Giappone. Il sacrificio è senza mezzi termini, eroico e definitivo. Elena, nella disillusione sente il bisogno di compiangersi sia pure accettando la propria condizione con remissività solenne. Raramente la religione l'innalza e l'amore vien mescolato irreverentemente alla fede. Daniele, com e Silla, è malato di sensualità. Ne è ossessionato anche se essa si presenta in modo sottile, si insinui nella sua anima prendendo le vesti di motivi ideali. Daniele rinuncia all'amore volendo affermare la superiorità dello spirito sulla carne.

Al Fogazzaro piacque questa sublimazione amorosa al di fuori della logica e degli schemi istituzionali del matrimonio. I due amanti si amano spiritualmente in attesa di ritrovarsi nell'aldilà, ma si tratta di un aldilà piuttosto mondano: "Vorrei che fosse ancor bionda là nel pianeta lontano" (p. 1286). 
Con il personaggio Elena il Fogazzaro ha voluto rappresentare un momento della sua vita, dramımatizzando gli elementi del contrasto spirituale ed assegnando al Cortis un valore simbolico.

In questo romenzo è caduta l'impalcatura romanzesca che aveva appesantito Malombra. L'assunzione psicologica di Daniele, la quale impedisce al protagonista di agire al momento opportuno e is modo logico e necessario, nasce dallo squilibrio tra idealismo e realismo. Elena rimane una delle più poetiche creature femminili del romanzo fogazzariano. Ella accarezza spesso i propri vagheggiementi che la inducono a vedere l'amore come tenerezza, la vita come consumazione di tenerezza e la morte come su tutto incombente. La sua rinuncia è eroica e al tempo stesso umana: un'anima nobile, dignitosa, desiderosa di abbandonarsi fra le braccia dell'uomo che ama, ma anche consapevole di dover ubbidire al dovere coniugale. Questa alternativa di attrazione e di fuga, che può essere contraddittoria nelle condizioni reali, non è mai contraddittoria quando si conduce alla legge universale dell'amore, che è legge appunto di fuga e di attrazione.

Il Fogazzaro ha rivissuto l'intreccio del romanzo con vivo e profondo interesse non volendo condurre i due amanti alla rinuncia ma, a seguito di una sua crisi spirituale, ha spostato l'epilogo amoroso e felice in un dramma di sofferenza e di passione. Questo forzato epilogo è quasi che una manifestazione astratta, una volonta estetizzante per cui ci sembra esatto quanto scrive Francesco Flora: "Mentre l'amore è disegnato con sapienza, il contrasto a questo amore, e il doverc a cui Elena si sacrifica, rinnovando fedeltà a un turpe marito, non hanno la necessaria adesione nella fantasia dell'artista".

\section{Il mistero del poeta (1888)}

Secondo il Fogazzaro, questo romanzo, (p. 1510-790) il terzo in ordine cronologico, dopo Malombra e Daniele Cortis, avrebbe dovuto essere l'espressione più alta della sua sofferenza amorosa, l'esaltazione del suo animo tormentato verso l'eterno. In Malombra l'amore era stato concepito in senso 
demolitore e diabolico; in Daniele Cortis, come dovere e deliberata rassegnazione.

L'opera non raggiunse in verità quella dignità letteraria che l'autore desiderava, essa è una esposizione debole e priva di colore, tutta pervasa di espresioni liriche e sentimentali, senza calore, senza fusione tra l'ambiente e la vicenda che in esso si incentra. $E^{\prime}$ l'opera, infatti, che più di ogni altra, viene sottoposta al giudizio severo della critica.

Il Fogazzaro riesce, come sempre, a tratteggiare felicemente, con arte e vivezza realistica, i tipi e le macchiette della provincia tedesca.

Lo stile si rivela molto diverso in relazione alle altre opere poichè in diversi punti, appare piuttosto disuguale, irreale, evanescente, mentre in diversi altri troppo preciso $e$ realistico.

L'autore ci racconta, in un alternarsi di prose e di versi, l'amore che egli senti per una bionda fanciulla straniera, di salute malferma, ma che venne distrutto dalla morte di lei.

L'opera, pubblicata nei primi mesi del 1888 , vorrebbe essere la narrazione quasi distaccata di questa passione giovanile, di questo "mistero" che il poeta per tanto tempo ha custodito segretamente nel proprio intimo, e che ora, nell'età matura, ripensa, come un sogno remoto, non per giudicare se stesso, ma quasi scettico, incredulo, così che può narrarlo addirittura ad una donna che lo interroga con uno certo desiderio di conoscere il suo "mistero". Lo scrittore narra la passione amorosa che egli ha provato per la dolce Violet Yves, figlia di un pittore inglese e lei stessa pittrice, che, rimasta orfana di entrambi i genitori, va a vivere a Norimberga, presso alcuni parenti della mamma. L'incontro tra il poeta e la bioda straniera avviene presso un albergo, nelle vicinanze del lago di Lugano. Il poeta rimane colpito dalla grazia sottile e dall'animo dolce della bella fanciulla, anche se un po' miope e alquanto claudicante. Dopo la scomparsa di Violet (muore proprio il giorno del matrimonio, stroncata da un collasso cardiaco), il poeta è sconvolto e vive tutto immerso nel ricordo di lei, allo stesso modo in cui Dante e Petrarca rimpiansero Beatrice e Laura. Questa ideale sopravvivenza di Violet, a tutti sconosciuta è il mistero del poeta. 
Violet è la primá straniera che noi abbiamo occasione d'incontrare in tutta l'opera del Fogazzaro. Violet è vista dal poeta in un'atmosfe.:a quasi irreale, in una luce di sogno. Forse l'opera può essere interpretata come spiritualismo e desiderio di evadere dalla realtà, o quale impossibilità di vivere la vita così corne $\mathrm{e}$.

La figura del protagonisia è sfumata, anche se l'autore si sforzi di presentarcela con la sua indole contraddittoria, ora incantato ed appassionato, ora egoista ed altruista, ora incredulo e leggero, desicleroso di vacuità e arciente di aspirazioni trascendentali.

Originale appare, invece, la rappresentazione della vita di provincia di una cittadina tedesca, con le sue consuetudini. Il Fogazzaro aveva visitato i luoghi descritti nel romanzo ed aveva, come era solito fare, preso appunti dalla realtà per riportarli, poi, nella sua opera. L'autore, però, si perde spesso nell'osservanza minuziosa dei suoi appunti turistici, caratteristica, questa, di tutta la sua opera.

\section{Piccolo mondo antico (1895)}

Indubbiamente possiamo affermare che Piccolo mondo antico (p. 2140-380) è il romanzo più sano del Fogazzaro, il capolavoro.

L'opera contiene il conflitto fra la ragione e la fede, rispettivamente rappresentato dai protagonisti Luisa e Franco: conflitto che sarà uno dei temi di riflessione dell'autore e che lo porterà a partecipare alle polemiche sul Modernismo.

Preoccupazione costante del Fogazzaro, durante la stesura, fu quella di scrivere un'opera bella ed anche buona; riuscì a comporre in sintesi artisicica le varie e contrastanti tendenze dello spirito, a liberarsi sufficientemente da preoccupazioni autobiografiche, evitando il difetto presente negli altri romanzi, dove $i$ dibattiti spirituali dei personaggi sono staccati dal contenuto poetico.

Nel romanzo, il dissidio religioso tra i due protagonisti è trattenuto nella sfera della modesta vita quotidiana che essi conducono; a smorzare un poco tale dissidio religioso contribuisce la rappresentazione del piccolo e chiuso ambiente di provincia. 
Per la prima volta lo scrittore abbandona sia l'ambizione di rappresentare passioni torbide e inquietanti, sia la tendenza a cercare personaggi eccezionali e lontani dalla realtà. Il romanzo si riduce ad un racconto sereno e tranquillo di piccoli avvenimenti, i cui protagonisti vivono la loro umile vita in una valle serena e tranquilla, la modesta Valsolda.

Caratteristica peculiare dei due personaggi è la fede dell'uno e la ragione dell'altro. Luisa che vuole vedere realizzato il suo ideale di giustizia in questo mondo e non in una vita futura, non riesce a comprendere il cattolicesimo vago ed incerto del marito. Quando piomberà sui due la terribile fine della loro figlia Ombretta, Luisa, che sembrava la più forte di carattere, si abbatte. Franco, al contrario, un tempo debole e incerto, troverà nella fede la forza necessaria per resistere alla sventura.

Tutta la vicenda si accentua sugli effetti che nell'animo dei protagonisti produce la morte di Ombretta. Alla fine, nell'anima di Luisa si anniderà la fede, mentre Franco comprenderà che la religione è vitia e non sogno e che è necessario guardare all'altro mondo artraverso questo.

L'amore non ha più, qui, come in altre opere del Fogazzaro, quella posizione di preminenza ossessiva. In Piccolo mondo antico i personaggi sono cari alla memoria dello scrittore, ma lontani dal suo dramma intimo, cioè dai vari dissidi della sua anima. Tutti i personaggi sono mirabilmente fusi nell'ambiente circostante, ne costituiscono la vera ed autentica voce: vi è perfetta rispondenza fra i personaggi coinvolti negli eventi e la natura nella quale essi vivono. Possiamo dire che $i$ luoghi rappresentati nel romanzo paiono animarsi nello svolgersi dei fatti, quasi soffrissero essi stessi il dramma dei protagonisti. Ovunque avvertibile è la presenza del lago, che diventa lo strumento del destino nella tragica fine di Ombretta.

In Piccolo mondo antico il Fogazzaro ha realizzato lo scopo che si era prefisso: dimostrare che il Cristianesimo non deve essere soltanto contemplativo, ma deve concretizzarsi nella vita stessa e che le opere, senza il sostegno della religione, restano sterili e fini a se stesse. 
La narrazione è rapida ed agile, senza digressioni prolisse che suscitino fastidio nel lettore. E' questo il momento particolarmente felice dell'arte fogazzariana.

\section{Piccolo mondo moderno (1900)}

Questo romanzo (p. 2875-3120) è il secondo di una tetralogia che comprende Piccolo mondo antico, Piccolo mondo, Il Santo e Leila. Dovrebbe essere la continuazione di Piccolo mondo antico, però ne è lontano sia artisticamente che moralmente. Infatti, dopo la parentesi serena di Piccolo mondo antico, in questo romanzo i problemi intellettuali dell'autore, impegnato ormai nella sua battaglia modernistica, turbano lo svolgimento, già umanamente debole, del racconto.

Il romanzo è ambientato in una piccola provincia che, pur non essendo indicata, mostra di essere la Vicenza dei tempi del Fogazzaro. La vita di questa ci viene offerta in un quadro gremito di motivi contingenti dai quali l'autore vicentino non è riuscito a sgomberare il campo della sua osservazione: osservazione senza dubbio acuta, ma povera di prospettiva e non abbastanza elaborata. I personaggi non acquistano mai personalità umana. Su queste figure senza ideali e senza nobili illusioni si appunta la satira e e lo scherno dello scrittore.

Il protagonista è Piero Maroni, figlio di Luisa e di Franco di Piccolo mondo antico. Dopo la morte dei genitori che tanto avrebbero potuto aiutarlo nella sua formazione spirituale, fu educato in un ambiente abbastanza severo, per cui nella solitudine sviluppo una natura al tempo stesso mistica e sensuale. Piero, come Corrado, riflette i travagli del suo autore, oscillando tra cielo e terra. Anche Jeanne Dassalle è tentatrice e purificatrice ad un tempo. Sposato ad una marchesina, di natura fredda, che non molto tempo dopo le nozze finirà in un manicomio, Piero conosce Jeanne, una donna piena di seduzioni fisiche e tuttavia restia a concedersi. Dopo tante riluttanze i due finiscono per essere travolti dalla loro passione e tuttavia, in una notte in cui Jeanne si sarebbe concessa, Piero riceve un telegramma annunciantegli che la moglie è in fin di vita e che desidera rivederlo. Ecco che la marchesina morente è vista da Piero in una luce nuova e 
meravigliosa ed egli risente il mistico turbamento provato da giovane. Alla morte della moglie decide di lasciare il mondo e dedicarsi ad opere religiose: siamo già al tema del Santo.

L'opera non è certamente tra le migliori, abbastanza monotona e torbida, eccetto alcune pagine di penetrazione psicologica e di drammatica animazione del paesaggio. Il corso degli avvenimenti viene spesso interrotto da innumerevoli elementi accessori ed anche $i$ protagonisti sono figure scialbe.

\section{Santo (1905)}

Questopera, (p. 3590-710) a parte i fattori erotici e sentimentali che vi confluiscono, rappresenta senza dubbio il lavoro di maggiore impegno programmatico.

Il programma l'abbiamo nell'incontro che il protagonista ha con il Pontefice. Questo incontro è il punto-chiave del romanzo e investe tre ordini di problemi: quello dei rapporti fra la Chiesa e il nuovo Stato nazionale italiano, quello dei rapporti fra la dottrina cattolica e le conquiste dell'imponente movimento scientifico determinatosi nel secolo XIX, quello di una riforma interna della Chiesa, vista, insieme, come ritorno alla carica mistica della rivelazione e come adeguamento delle strutture ecclesiastiche alle esigenze della società contemporanea."

Queste esigenze del protagonista, o meglio, queste componenti dell'ideologia del Fogazzaro, si riallacciano, in particolar modo, al romanzo Daniele Cortis che possiamo definire como il manifesto politico dell'autore. Anche Daniele Cortis, il protagonista, dimostra il suo disgusto per la realtà postrisorgimentale e, fervoroso, prende in mano le questioni sociali. Le sue idee, Daniele le espone nello schema del discorso che dovrebbe pronunciare alla Camera: polemica contro la corruzione del regime parlamentare e richiesta di una monarchia assoluta, non per eliminare la libertà, ma per disciplinarla; polemica contro le tendenze anticlericali e massoniche, e riconoscimento della funzione moderatrice del sen- 
timento religioso. C'è insomma, in Daniele Cortis, la proposta della costruzione di uno stato moderno e di una riforma della Chiesa, proposte queste che rincontriamo più accuratamente collocate nel romanzo II Santo.

Il protagonista, all'inizio del romanzo, ci appare fragile $e$ in conflitto tra ragione e senso. poi lo incontriamo in veste di santo, fervoroso nella propria opera riformatrice, ostacolato e non compreso da molti, infine lo vediamo soccombere e rassegnarsi.

Il rinnovatore è quel Piero Maironi di Piccolo mondo moderno che, chiamato al capezzale della moglie morente, risente il mistico turbamento che aveva sentito da giovane $e$ decide di lasciare il mondo e dedicarsi ad opere religiose. Lo ritroviamo all'inizio del romanzo, nel convento dei Benedettini a Subiaco, dove è diventato Benedetto e dove si purifica, attraverso la penitenza e il lavoro manuale. Guida spirituale di Benedetto, in questi momenti difficili del noviziato, c̀ il buon padre Clemente nella cui casa si raccolgono filosofi e teologi che desiderano il rinnovamento della Chiesa.

Spinta dal desiderio di conoscere la vera identità dell'umile frate, nel dubbio che si tratti del suo Piero, Jeanne va a vederlo e lo riconosce. Benedetto, tuttavia, disillude la sua amante di un tempo, sia pure promettenciole che, in punto di morte, la richiamerà. Dopo questo episodio Jeanne scompare e Benedetto viene mandato in un paesetto situato sui monti che circondano Subiaco. Qui, in breve tempo si fa la fama di santo, ma anche molti nemici. Cacciato dal convento, nell'abbanciono e nella miseria spirituale e materiale, Benedetto sente una voce che lo chiama ad un'alta missione: quella di andare a Roma. Nell'umile quartiere del Testaccio cgli inizierà il suo nuovo apostolato.

A Roma il Pontefice mostra interesse alle sue idee di riforma della Chiesa ,ma anche qui i clericali che gli sono contrari si adoperano per farlo soccombere.

Il romanzo è alla fine: Benedetto, arso da una violenta febbre è prossimo a morire. Riappare, a questo punto, Jeanne che, al corrente dell'imminente morte del suo antico amore, mette alla sua disposizione una villa. L'agonia è lunga solo poco prima che Benedetto esali l'ultimo respiro, giunge 
Jeanne la quale, abbandonato l'orgoglio, bacia il Crocifisso che egli le porge, pervasa da un improvviso fervore religioso.

Jeanne qui ci appare dolorosa e lacrimante, con tutta la sua capacità di abnegazione e di sacrificio, una Jeanne diversa da quella di Piccolo mondo moderno dove era il simbolo della bellezza che seduce ed incanta. Benedetto resta la scialba figura dell'opera precedente. La sua conversione non è stata frutto di un lungo travaglio, ma è giunta quasi per miracolo. In lui riscontriamo la divergenza tra la grandezza della missione e l'impossibilità umana di risolverla; tuttavia egli assunse una fondamentale importanza perchè vi si possono riscontrare le debolezze ed i compromessi del Fogazzaro stesso, la sua biografia spirituale nel senso ampio di ideali e principi politici, morali e religiosi.

\section{Leila (1910)}

Con Leila (p. 4005-290) si affronta il problema del realismo romantico in cui il Fogazzaro cerca di riallacciarsi alla tradizione veneta e al diffuso realismo del secolo ottocento.

Il romanzo si svolge, fin dall'inizio, come commedia amalgamata con personaggi ritratti dal reale quasi bozzettisticamente.

L'elemento umoristico e realistico è dato dalla descrizione di personaggi minori; il tono narrativo diventa man mano sentimentale e lirico in rapporto all'importanza dei personaggi.

Il romanzo fu pubblicato a Milano, l'anno prima della morte del Fogazzaro. Il protagonista è Massimo Alberti, discepolo di Benedetto, il santo nel romanzo omonimo.

Massimo, travolto dalle lotte dottrinali, perde la fede. Intanto si innamora di Leila, che vive nella casa di Marcello Trento, padre del defunto fidanzato. Marcello ha adottato Leila e vuole che ella sposi il più caro amico del figlio: Massimo. A queste nozze si oppongono l'arciprete e il cappellano poichè non tollerano la posizione polemica che Massimo ha assunto nei riguardi del clero. Anche il padre vero di Leila è contrario a queste nozze, avido di mettere le mani sui beni della figliuola, unico erede del patrimonio del signor Marcello. 
Leila, temperamento carico di contraddizioni, dapprima lo respinge, infine peró, persuasa della sofferenza di Massimo, corre a raggiungerlo. Massimo troverà nell'amore di Leila, la spinta sufficiente per il ritrovamento della fede religiosa.

Il romanzo dette inizio ad una serie di giudizi contrastanti. Alcuni videro in esso un allontanamento del Fogazzaro da quel desiderio di riforma e di rinnovamento della Chiesa, propri dei modernisti. Altri vi videro un ritorno alla fede tradizionale, sebbene il Fogazzaro polemizzasse con una satira pungente contro il clero e i clericali. Fu questo il motivo per cui il romanzo fu messo all'Indice.

L'opera è artisticamente mancata; appesantita dalla polemica religiosa, non si lega alla storia d'amore che dovrebbe essere il motivo conduttore di tutto il romanzo. Gli stessi personaggi, ed in particolare, Leila, rivelano la stanchezza dello scrittore che non fa che ripetere vecchi motivi e non sempre convincenti.

Leila ha il suo animo tormentato come quello di Jeanne e Marina, ma l'orgoglio non le consente l'abbandono totale alla passione e la sua sensualità sfocia in un romanticismo sdolcinato, ricco di compiacimento estetico.

Leila, ultimo romanzo del Fogazzaro, avrebbe dovuto essere la ritrattazione, un libro di chiarificazione del sentimento religioso dell'autore, una difesa contro le accuse di molti rivolte all'opera precendente. Leila, in realtà, è il proseguimento de 11 Santo in chiave polemica.

\section{CONCLUSIONE}

Le caratteristiche fondamentali della poetica del Fogazzaro sono: la rappresentazione della natura che sappia cogliere in essa il rapporto fra i sentimenti umani e le voci occulte delle cose, con un conseguente e vago senso del mistero; la rappresentazione di drammi intimi di creature d'eccezione, appartenenti alle classi alte della società, colte, raffinate, sensibili; la rappresentazione di ambienti confortevoli e spesso mondani; il ritratto dal vero di personaggi comici (che servono a dare maggior risalto ai drammi morali e sentimentali 
dei protagonisti e, nello stesso tempo, ad allegerire la narrazione); la rappresentazione idillica dei rapporti sociali; la salvaguardia delle istituzioni della morale borghese monostante che si giunga quase sull'orlo dell'abisso; una struttura linguistica che si adatti al gusto medio, né troppo veristica, né troppo aulica e letteraria."

\section{RESUMO}

As obras de António Fogazzaro descambam no autobiografismo que nāo é confissão dolente e sim representaçāo das perplexidades, das experiências morais e religiosas do autor. Nelas há uma proposta religiosa, como também uma proposta politica, as quais, paridas de uma conjunçăo hibrida do ideal e do real, revelam-se contaminadas e confusas.

Contudo, esses "principios" fogazzarianos, sob uma perspectiva diversa enquadrada nos tempos e na realidade histórica do momento, dão validez ao propósito de predicação religiosa e politica.

Os protagonistas dos romances, seres fora da norma, são, "lato sensu", opacos, sofisticados e desligados do contexto poético por estarem assoberbados pela presença do autor.$O$ autor, de fato, repropōe o protagonista do romance anterior com umas poucas variaçరes e com nome diferente: 0 personagem principal nada mais é que o personagem no qual o autor quer se representar com todos os seus conflitos, os seus contrastes e entre o céu e a terra.

\section{REFERENCIAS BIBLIOGRAFICAS}

1 CROCE, Benedetto. La letteratura della nuova Italia. Bari, Laterza, 1947. $5 \mathrm{v}$.

2 DONADONI, Eugenio. Antonio Fogazzaro. Napoli, Perrella, 1913. $215 \mathrm{p}$.

3 FLORA, Francesco. Storla della letteratura Italiana. Milano, Mondadori, 1940. $5 \mathrm{v}$.

4 MOMIgliano, Attilio. Elzevirl. Firenze, Le Monnier, 1945. 470 p.

5 NARDI Plerc. Vita e opere di Antonio Fogazzaro. Mllano, Mondadori, 1931. $12 \mathrm{v}$.

6 SALINARI, Carlo. Mitl e conscienza del decadentismo Itallano. Milano, Feltrinelli, 1970. $288 \mathrm{p}$.

10 Vedcre BALINARI. D. 200-4. 